\title{
What stops most large 'customer relationship managers' optimising their customer communications programmes? Part 1
}

Received (in revised form): 20th September, 2002

\author{
Julian Berry \\ is currently a member of the Sophron Partnership and boasts extensive experience in many aspects of customer relationship \\ management. During his tenure as Managing Director of Berry Consulting (1990-99), he played a key role in the development \\ of the pioneering financial segmentation system FruitS and the customer decisioning software Contract Planner, the latter \\ winning gold in the Royal Mail/DMA awards. \\ Prior to Berry Consulting, and its subsequent sale to Acxiom Ltd in 1999, he had been Head of Direct Marketing at TSB \\ Bank and Head of Marketing Services at Centre File. He is a frequent lecturer and writer in journals such as the Journal of \\ Database Marketing.
}

\begin{abstract}
This is intended to be the first of two papers that will attempt to unpack some of the issues that surround the broad and complex question of 'optimisation' in the context of managing customer communications through time, to large cohorts of consumers, who, because of some historic transactional relationship, may be described as customers.
\end{abstract}

\author{
Julian Berry \\ Sophron Partner, \\ The Studio, 2 Dover House \\ Road, \\ London SW15 5AU, UK \\ Tel: +44(0) 208878 9350; \\ e-mail: \\ julian.berry@btclick.com
}

\section{INTRODUCTION}

The optimisation issues can be split into three high-level categories of objective, measurement and means; in other words, what are marketers trying to achieve, how is it measured and how is it achieved. Part 1 is concerned with objectives and their measurement, while Part 2 will cover the means.

Because of strong industry focus on the means, ie the technology and tools to use in customer communications, there has been less interest in the reasons for undertaking them, and the means by which their true impact can be evaluated. And if the focus on the objectives is not correct, then it is self-evident that the wrong tools may be designed and built to serve them.

As the author has been a working consultant in this field for well over ten years, he has had the privilege of insight into the internal operations of a large number of UK and foreign clients, most with many millions of customer relationships to look after; in each case he has been subject to confidentiality agreements so this paper necessarily abstracts from these learning experiences rather than instancing actual case studies. The reader may, however, be assured that the abstractions are in every instance based on reality, and because of cultural similarities between many businesses these realities have a tendency to be repeated.

It is the supposition here that, in the context of mass customer communications, it could fairly be said that the cart has often been leading the horse, while the driver has been preoccupied with justifying the cost of 
both, and losing focus on where they are supposed to be going in the first place. These papers will attempt to explain why.

\section{THE KEY 'ISSUES' OVER OPTIMISATION OF CUSTOMER COMMUNICATIONS OBJECTIVES}

It is obvious that mass communications not only have a hugely diverse range of effects on their individual recipients, but that the nature of the impact of standardised communications is greatly affected by how they are targeted and their arrival timed. The paper starts therefore with some examples of where failure to set the right objectives had led to suboptimal results. It is hoped that these will illustrate some of the issues in question.

The first example is of cases where the business has demanded such response volume results in the short term that, as a result of consequent over-communication, and often repetitive and irrelevant messaging, customers have taken to opting-out in numbers that are leaving up to a third of them incommunicable. Whereas it used to be a norm that around 5-10 per cent of a customer base would have a 'don't-mail' marker, the numbers the author now encounters have risen steeply above that, and frequently over 20 per cent. And when looking at levels of opt-out it is important to distinguish between the cumulative and the recently-recruited cohorts, where the numbers can be double those of more established customers. It is also often the case that the younger customers will use the opt-out facility more frequently. Clearly, the reasons behind don't mail markers may be several, and an opt-out selected at an application stage has a quite different meaning to one applied mid-way through a relationship.

Nevertheless the overall message conveyed by the opt-out sector of the customer base is powerful and simple 'Given the way you are communicating with me today we would rather not hear from you in future'. As strong a negative response to the corporate brand as can be imagined. So, in objective-setting terms, the maintenance of the brand value through time has been regretfully ignored.

The second example is of cases where the business has assumed that by focusing on the return on investment (ROI) from its communications expenditure that it will necessarily optimise the result in terms of contribution.

This scenario occurs extensively where getting the most out of a limited budget is the key objective. It can even lead to such perverse results as a company preferring to do deals with third parties to market to their customer base, and relying on commission deals, rather than spending their own budget, because the return on the small amount of cost incurred when providing customers for others to exploit shows an exceptionally high ROI ratio. More commonly, it favours the campaign or product-led focus rather than one relying on developing customer value exchange. ROI is a ratio, quantifying the return made from $\mathrm{EX}$ of communications. It can be thought of as $[\mathrm{P} \times \mathrm{V}-\mathrm{C} / \mathrm{C}]$ where $\mathrm{P}$ is the response and conversion propensity, $\mathrm{V}$ the value exchanged with the customer after conversion, and $\mathrm{C}$ the cost of undertaking the communications. The classic ROI approach is rewarding communications efficiency, often at the expense of effectiveness in terms of delivering customer value.

This is a key distinction and will be revisited frequently in these two papers. It does, however, have some merit; the campaign with the higher ROI will have more chance of getting budget and being included in a programme than one with 
a lower ROI, even though both may be comfortably profitable.

The third example concerns cases where the business will impose no limit or control on the level of communication as long as each campaign is in itself profitable. In this case, apart from the opt-out effect, another force comes into play. Anything with an ROI above ' 1 ' is acceptable, the customers are blitzed and response rates drop.

When a start is made on diagnosing what is wrong with this approach in terms of objective setting it is necessary to look at the case of the individual customer; instead of the customer being made just that offer that he would most prefer at that time, he is being made any number of offers where, because he has been categorised by the business and not by himself (eg through the prior use of permission marketing techniques) as being a member of a group that overall provides a positive contribution, he can be profitably communicated with.

So, the objective issue is one of targeting maximum communication contribution assuming that one campaign has no impact on the results of another, rather than optimising customer value development.

The fourth scenario is where a customer-level strategy has not been imposed in front of a communications one. In these cases large cohorts of unprofitable customers continue to receive communications which, at a campaign level, may appear to be profitable, but which do no more than preserve unsatisfactory relationships. It is a truism that most customer bases contain unprofitable segments, and for banks and credit card companies this can be as high as a half, but few businesses measure profitability successfully at an individual customer level; consequently they cannot impose a customer management strategy prior to a communications one. The business objective required is to determine whether a continuing relationship is or could be beneficial before deciding how it is to be conducted. Admittedly, this is complex where the benefit of the relationship may be longer term, and it is currently loss making, but not to start working out how to employ customer management techniques is worse than unfortunate.

The fifth and final example is of cases where little or no attempt has been made to manage communications density and intervals optimally. This is, in a sense, a subset of the third case, but focused on timing. Anyone who has conducted live experiments with the timing of communications finds it a fascinating experience, as there are optimal timing intervals for any proposition (when judged by response) and for any customer segment optimal densities when judged by contribution. In reality, however, few companies understand either. This common issue normally occurs because the knowledge and tools tend not to be available to facilitate the process; few companies would not wish to include managing density and intervals among their objectives, but they have not committed themselves to the live experiments to find out the impact of alternative densities and intervals, and their communications management tools tend to be better at applying interval rules than at optimising density through time.

\section{SETTING COMMUNICATIONS OBJECTIVES}

It may help to propose that not only multiple objectives are required but also a hierarchy within them so that decisions about managing communications to individuals may be optimised. 
Table 1

\begin{tabular}{lll}
\hline & ROI objective & Customer value objective \\
\hline Budget constrained & Red zone & Brown zone \\
Customer constrained & Blue zone & Green zone \\
\hline
\end{tabular}

From the illustrations above it can be seen that there are at least two levels of objectives to consider:

- strategic, eg only investing in extending relationships with those the company wants to do business with while above all else maintaining the brand's esteem for them

- financial orientation, eg focusing on optimising communications ROI or on optimising customer value exchange.

Only at this stage can a start be made on resolving the perennial problems of what to offer to whom, how often and when. Yet most businesses begin here.

The strategic decisions about those to do business with require an understanding of current and potential customer profitability at either an individual customer level, or for a segment. Once this is known then objectives can usually be simplified down to grouping customers into desirable, unproven and undesirable and setting appropriate longer-term communications strategies for each.

Setting brand objectives is harder in the sense that customer research never appears to get to the bottom of what customers really think about the customer communications they receive.

A major UK medical charity once wrote to its donors asking them what communications they wanted, about which conditions, for which groups of sufferers, and through which channel. Rational stuff, but the outcome was that the donors voted for minimum communications on any topic and maximum expenditure by the charity on its declared medical objectives. The donors had not linked the need of the charity to raise money from them with their expenditure on donor communications.

In another case, a credit card company with high direct mail and outbound tele-marketing levels and above average customer opt-out rates, tried to obtain feedback from its non opt-outs about its communications programme. Curiously, virtually no-one responding expressed any dissatisfaction about the type or level of communications which they were receiving. The problem was how to direct the question at the dissatisfied customers rather than the complacent.

Hence for brand issues the objectives most probably have to be set in terms of measurable symptoms, such as allowable levels of opt-out, complaints, account terminations etc, rather than their underlying causes such as levels of dissatisfaction with certain types of communications activity.

The financial objectives can be better understood by setting up a typical consultant's matrix as shown in Table 1.

This matrix is useful in helping to understand where an organisation is in terms of its development, and what tools and measurement systems it needs to achieve their financial goals.

\section{Red zone}

This is the majority experience where getting the best ROI from campaigns, at the minimum cost per sale, is the principal objective. It is suboptimal in 
that it will not optimise on the latent value potential of the customer base, and it will not provide each customer with a brand-enhancing experience. (Instances have even been seen where switching the objective from campaign ROI to cutomer contribution has improved the overall contribution achieved from a given budget by as much as 20 per cent or more.)

The key characteristics of companies in this zone are:

— budgets are owned by product management

- communications opportunity costs are not recognised

- interactions between communications are ignored.

The key tools and processes required to succeed in the red zone are:

- campaign-level response targeting models

— inter-campaign budget optimisation

- campaign selection sequence optimisation

— outbound-process efficiency.

\section{Blue zone}

This is the area where companies are still ROI driven, ie they wish to optimise on their return from the communications budget, but they do so by looking at the investment in and return from individual customers rather than just in communications.

It is only suboptimal in the sense that ROI has been selected, rather than customer value, as the objective.

The key characteristics of companies in this zone are:

- they have developed an understanding of individual customer value and how to measure changes in it
- budgets are managed from a customer value ROI perspective

- budgets are distributed optimally across all channels including sales off service

- processes are employed to link communications to customer value exchange

- understanding exists of how to optimise communications through time.

The key tools and processes are:

— introduction of "next best activity" individual customer-level decisioning

- single customer view linked to channels

- use of a planning simulator

- sophisticated customer insight tools including triggers, propensities and segmentations.

\section{Brown zone}

In this area, the objective is to use the given communications budget to optimise the level of customer value achieved. The key difference compared with the blue zone is that the emphasis is on the level of customer value or contribution achieved, rather than ROI. It is suboptimal only in that it is not recognising the latent customer value in the customer base as the final constraint, and is instead budget constrained.

The key characteristics, tools and processes of companies in this zone are virtually the same as those in the blue zone. Decisions about customers, however, are based on customer contribution not ROI.

\section{Green zone}

In the green zone the objective is to maximise customer contribution through 
time, the ultimate state for those managing customer communications.

The key differences compared with the blue and brown zones are that budget is made available as long as the contribution is positive, and that the only criterion for its use is its impact on longer-term customer contribution development.

Again, the characteristics, tools and processes are otherwise similar to companies in the blue or brown zones.

It will have become clear to the reader that the big challenge is to move out of the red zone; the subtleties of being in the blue, brown or green zones are only important once one has arrived there, in that they reflect either the constraints under which the business works, or the goals of management.

Part 2 of this paper will concentrate on the tools needed to deliver customer-level decisioning, the key requirement for moving out of the red zone, and the constraints that most businesses will need to overcome to get there.

\section{WHAT NEW APPROACHES TO MEASUREMENT AND EVALUATION DO COMPANIES NEED TO ADOPT WHEN MOVING OUT OF THE RED ZONE?}

The key characteristics of a suitable measurement system are that it addresses changes to customer value, which can then be expressed either absolutely for zones brown and green, or as a ratio compared to customer communications investment for zone blue.

Measuring changes to customer value presupposes an understanding of value itself, and a company's ability to measure it at an individual level.

Because customers are subject to many influences other than the communications sent to them by businesses, a control group not receiving these communications has to be maintained in order to get to the bottom of this.

With a control group customer contribution can be summed and then averaged at the start and end of a period and consequently changes can be measured. In many businesses the communications effect is a much smaller element in value development than other factors, eg consumer confidence or interest rates. Also, communications may substitute for other activities such as retail effort, and hence their net effect has to be understood after possible cannibalisation. Counting responses and attributing a net present value to sales made as a result will never get to the bottom of factors like this.

So, what approach should be taken to customer value itself? From the author's experience successful approaches focus on measuring actual value delivered in the period in question, rather than relying on predictions of future value.

Figure 1 may help to explain how to achieve that. (C stands for individual customers, and $\mathrm{P}$ for the products they hold). It shows typical product ownership patterns per customer through time. Value is delivered through either product purchase (retail) or product ownership (services). These events need to be recorded on the customer database and then translated into value exchanged.

For every separate industry there are specific ways of calculating value, and for each company vagaries in the level of detail recorded, particularly at the level of costs per customer, which often have to be averaged, or applied at a segment level.

The objective, however, is to achieve a continuous record at an individual customer level of value delivered through time. From this it is then possible to start to track changes 


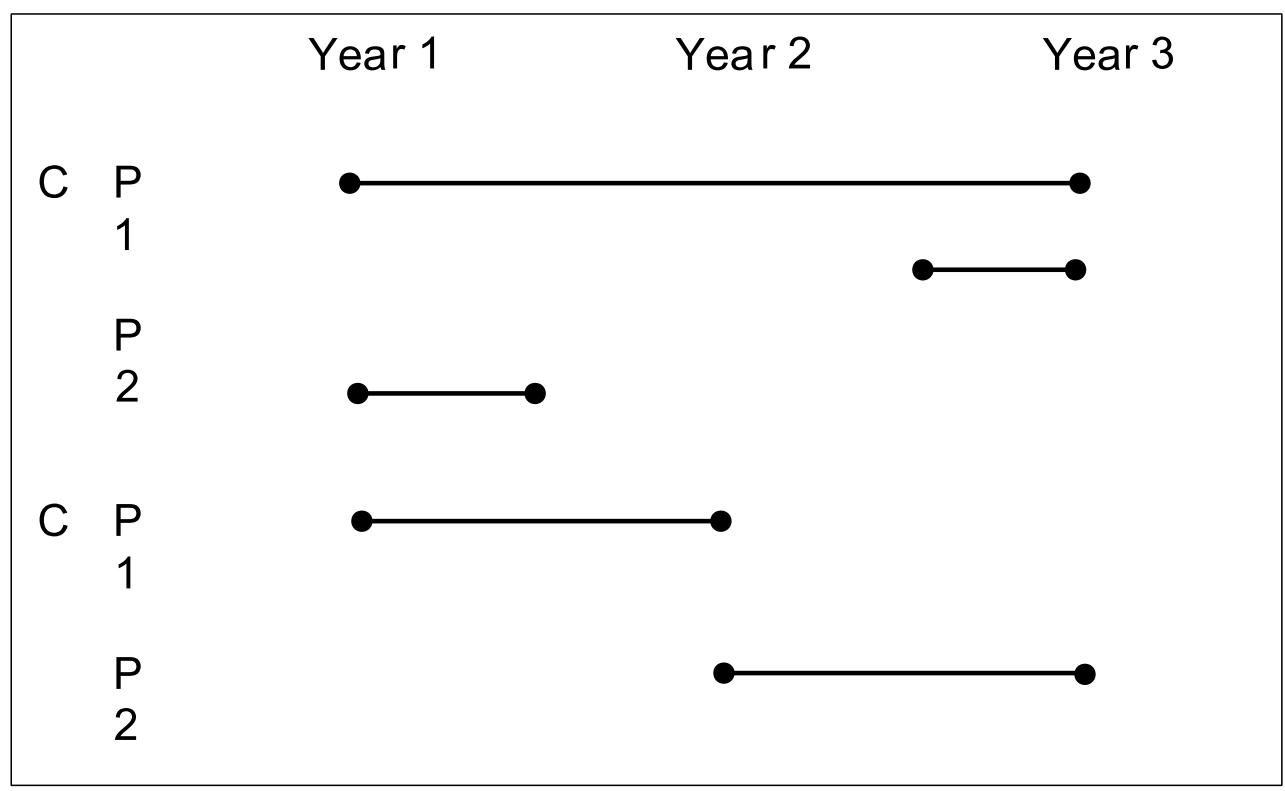

Figure 1

in the level of value delivered and hence the true measure of customer communications activity.

\section{INTERIM CONCLUSIONS}

So far it has been shown that many, or possibly most, companies fail to optimise their customer communications owing to their pursuit of the wrong objectives, ie return on campaign investment rather than increase in longer-term customer value. They remain locked in the red zone and ignore the potential for introducing a customer-level strategy.

To switch objectives requires, however, some fundamental changes to their measurement criteria, processes and tools. This paper has explored so far how the measurement criteria can be designed, and Part 2 will look at the tools and processes companies need to introduce. 